\title{
Global inventory control in an academic hospital
}

\author{
Nico Dellaert ${ }^{\mathrm{a}, *}$, Erik van de Poel $^{\mathrm{b}}$ \\ ${ }^{a}$ Erasmus University Rotterdam, P.O. Box 1738, 3000 DR Rotterdam, Netherlands \\ ${ }^{\mathrm{b}}$ BAZIS, Schipholweg 97, 2316 XA Leiden, Netherlands
}

\begin{abstract}
During a recent study concerning the global inventory control in an academic hospital we faced the problem of a hospital management that wanted a very simple inventory control model, with good performance. It was required that the members of the purchase department could understand the model and also that the model's control parameters could be determined in a simple way, because of the database environment of the inventory control system. Since most items have a joint supplier and the orders for a certain supplier are always placed on the same day of the week we extended an EOQ model to a so-called $(R, s, c, S)$ model, in which the values of the control parameters $s, c$ and $S$ are determined in a very intuitive way. To our surprise, the performance of this rule was comparable to that of a rule in which the control parameters were determined in a more sophisticated way.
\end{abstract}

Keywords: Global inventory control; $(R, s, c, S)$ model

\section{Introduction}

In the public health sector in the Netherlands the production economics has been neglected for a long time. Especially in the hospitals there was not much need for cost reduction, but this has changed drastically in the last years. Nowadays, there is much more interest in the actual costs of a patient, but also in the purchasing costs and holding costs of the hospital's inventories. At the start of our research we noticed a number of problems in the logistic field: the organisation, logistic procedures, knowledge of costs, missing inventory and the effectiveness of the inventory rules. The

* Corresponding author. inventories in a hospital can be divided in global inventory, which is stored in a number of large warehouses and which is controlled by the Purchasing Department, and the local inventories, spread over the various hospital departments and operating rooms, which are locally controlled. In this paper we will focus on the control of the global inventory in the Academic Hospital Rotterdam. The situation in this hospital is believed to be typical for most larger hospitals.

In the late seventies an administrative system called VOBE was developed for about fifty of the largest hospitals, including all academic hospitals, which had to take care of the global inventory control. This system is still in use, although with slight modifications. In VOBE a record is kept for every item that is stored in a global warehouse. 
This record includes, among other things, the total monthly demand of the local departments over the last 12 months, the product price, the ordering costs, the supplier's code, the last 6 delivery times and also a number of control parameters: the order level and the order quantity, both expressed in an integer number of weeks and also a so-called canorder percentage. For the can-order level we add a percentage of the order quantity to the order level. If the inventory of one of the items of a supplier drops below its order level, all other items of the same supplier for which the inventory is below the can-order level are also ordered.

From this description it is clear the VOBE system allows the hospital to use a type of $(R, s, c, S)$ inventory rule (see, for instance, [1]). However, the hardware of this administrative multi-user system does not allow complicated or time consuming numerical calculations. It is also a strong wish of the purchasing department to have an understandable algorithm for the determination of the $s, c$ and $S$ value of each item. A large difference between a hospital and companies in the production area is the non-technical background of the hospital workers (there are no engineers!). Therefore, a simple inventory rule is more important than it would be in technical companies. In this paper we will first discuss the current use of the inventory control system. In Section 2 we will also discuss the relevant cost structure. Section 3 contains the description of the proposed simple method. In Section 4 we describe some theoretical alternatives, especially a method proposed by Federgruen et al. [2], and compare the costs of this method and the simple method for some examples. Finally, in Section 5 we compare the various cost components and the service level for the new method and for the old method in a simulation study and we describe the results of the implementation, followed by our conclusions.

\section{Initial situation}

In this section we will describe the use of the inventory control system in the Academic Hospital Rotterdam in the initial situation. Basically an $(R, S, S)$ rule was followed for every individual item.
Table 1

Cost comparison between current and EOQ strategy

\begin{tabular}{lcc}
\hline & Current strategy & EOQ strategy \\
\hline Total costs & 222.638 & 133.067 \\
Holding costs & 90.612 & 66.348 \\
Order costs & 132.026 & 66.348 \\
Number of orders & 476 & 503 \\
\hline
\end{tabular}

The performance of such a rule depends on a number of elements. The length of the review period $R$ is important, as well as the values for the order level $s$, the can-order level $c$ and the order-up-to level $S$, but perhaps the most important thing is the way in which purchasers treat these levels. Until now the can-order level has never been used. At the start of VOBE, parameter values were set for the order level and order quantity of each item. This has been done for some hospitals together, where the order quantity was based on the EOQ rule. Although demand sometimes changed drastically, most of these quantities (expressed in weeks) did not change for more than a decade. If the can-order level is not used, the EOQ rule usually offers a good performance. We applied the EOQ rule on the items belonging to the group of printed matter, for which the external ordering costs vary from 0 to $400 \mathrm{Dfl}$. and for which we set the internal ordering costs to $25 \mathrm{Dfl}$. and the holding cost rate to $25 \%$. If we compare the EOQ results with the order quantities in $\mathrm{VOBE}$ we find the cost figures shown in Table 1.

In Table 1 we observe that both the total inventory costs as well as the total ordering costs are considerably less if we use the EOQ strategy instead of the current strategy. Striking is that despite a larger number of orders the total order costs decrease, because expensive orders with large external costs are avoided. In Table 2 we compare the number of orders per year for the items of the printed matter group. For each interval we give both the current number of items for which the average number of orders per year is in this interval and the number of items according to the EOQ strategy. From the second table it is clear that a proper updating of the order quantity has been neglected for a long time. 
Table 2

Order frequencies for current and EOQ strategy

\begin{tabular}{lcc}
\hline Orders per year & Current \# items & EOQ \# items \\
\hline $0.1-0.2$ & 18 & 93 \\
$0.2-0.33$ & 26 & 88 \\
$0.33-0.5$ & 21 & 74 \\
$0.5-0.67$ & 33 & 45 \\
$0.67-1$ & 89 & 48 \\
$1-1.5$ & 160 & 23 \\
1.52 .5 & 99 & 32 \\
$2.5-5$ & 13 & 33 \\
$5-26$ & 1 & 24 \\
\hline
\end{tabular}

If we compare the order levels (in weeks) with the delivery times of the items, we may expect a strong correlation. However, the VOBE order levels show a similar deviated pattern as the VOBE order quantities. The VOBE order levels are used rather strictly: if the inventory of an item drops below its order level, VOBE automatically generates an advice to order. Order advices for the same supplier are collected and once a week the items are ordered. Different from the order levels, the order quantities that are advised by VOBE are quite often disregarded by the purchasers. Usually the purchasers use the previous order quantity or argue that "according to their intuition the amount should be less or more than advised" and only very seldom there are good arguments for this, such as a quantity discount or a special price offer. Because of this behaviour, it is rather difficult to determine the exact total costs in the initial situation. Therefore, we will also use the size of the last six orders of an item as well as the recorded VOBE levels for making our comparison.

The global warehouses in the hospital can be divided into two parts: the sterile disposable warehouses (SDW) and the (non-sterile) central warehouses $(\mathrm{CW})$. In the sterile disposable warehouses all kinds of sterile medical items are stored, such as sterilized gauze, medicated cottonwool, catheters and surgery gloves. The central warehouse contains for instance items used for cleaning, printed matter, household ware and stationery. Because of the nature of the stored goods, the tenability of items in the SDW is less than those stored in the CW. This will be expressed in one of the components of the holding costs.

We split up the holding costs in three parts and for simplicity we suppose that the costs are always proportional to the product value. The first component is the interest rate or opportunity costs: this is set to $8 \%$ of the product value for both SDW and $\mathrm{CW}$ items. Because of the sterility needs, the relative housing costs of the sterile disposable warehouses are slightly higher than of the central warehouses: 12 versus $11 \%$. The third component contains the superfluous goods and the items that are no longer fit for use. For the SDW, this rate was estimated to be $9.5 \%$ of the product value and for the $\mathrm{CW} 6.5 \%$. Thus, the total holding costs rate is $25.5 \%$ for the items in the central warehouses and $29.5 \%$ in the sterile disposable warehouses.

Instead of working with imaginary shortage costs, the hospital management preferred the use of service rates: for the SDW $99 \%$ of the demand must be available directly (usually this meant within one or two days) and for the CW $97 \%$. The rate for the SDW is higher because of the medical use of most of its products.

For some of the stored items, especially for the printed matter, there are external ordering costs. For all items, internal ordering costs have to be made, not only at the Purchasing Department, but also at the Financial Department and by people working in the shipping area. Although part of these costs are more or less fixed, we will use the absorption costing method of Horngren and Foster [3] in which the total internal ordering costs are assigned proportionally over all orders. Thus, the internal ordering cost for an order is set to $D f$. 22.61. Every additional order from the same supplier leads to extra costs of $D f l .6 .36$.

\section{The inventory rule}

In this section we will derive a simple inventory rule, by which we can determine the values $s, c$ and $S$. We suppose that the value for the review period $R$ is given. For a certain item we might observe the following situation for a given level of $s, c$ and $S$ (Fig. 1). 


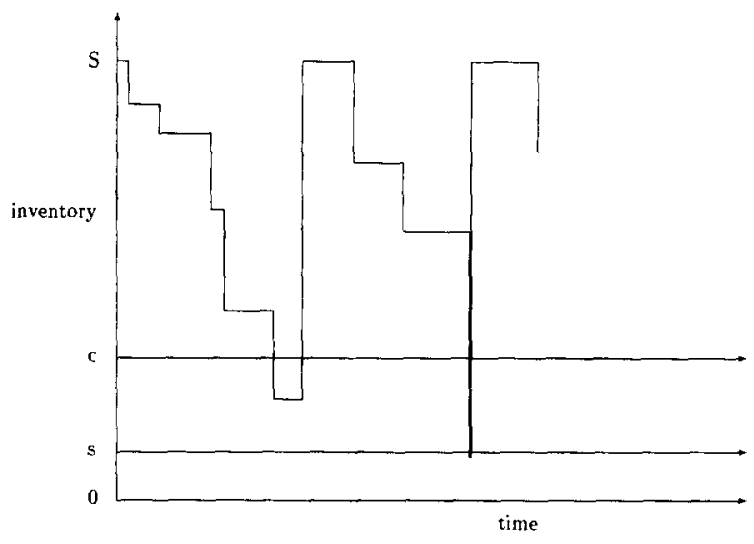

Fig. 1.

Now let $S=s+Q, c=s+X, v$ the unit price, $r$ the holding cost fraction and let $D$ be the average demand per time unit. Furthermore, let $1-P_{s}$ be the probability that the item is ordered before the inventory level reaches the $s$-value. For our analysis we consider the inventory level during one reorder cycle. First we will determine $X$ and $Q$ values for a fixed $s$-level. Therefore, we will not include the inventory below the $s$-level in the total relevant costs. If we assume that the ordering level is either $s$ with probability $P_{s}$ or uniformly distributed on the interval $(s, s+X]$ with probability $1-P_{s}$ then the expected total relevant inventory amount during the reorder cycle turns out to be

$P_{s} * Q * Q /(2 D)+\left(1-P_{s}\right)\left(Q^{2}-X^{2} / 3\right) /(2 D)$.

If the item is ordered before the $s$-level is reached, the only ordering costs are $a$, otherwise additional ordering costs have to be made. These additional costs $A$ can be shared with other items from the same supplier that reach their order level during the same review period. Suppose that on average $F$ other items reach their ordering level during a period of length $R$. Then the expected ordering costs per cycle for our item are given by

$P_{s} *(a+A /(1+F))+\left(1-P_{s}\right) * a$.

The expected length of a reorder cycle is given by

$P_{s} * Q / D+\left(1-P_{s}\right) *(Q-X / 2) / D$.
Thus for given $s$ the total relevant costs are given by

$\frac{a D+P_{s}\left(A D /(1+F)+Q^{2} v r / 2\right)+\left(1-P_{s}\right) v r\left(Q^{2}-X^{2} / 3\right) / 2}{\left(P_{s} * Q+\left(1-P_{s}\right) *(Q-X / 2)\right)}$.

We cannot simply find the minimum value of this cost function by taking the derivative and setting it to zero, since the value $P_{s}$ and the values of $X$ and $Q$ are strongly related. Therefore, we will first consider these relations. For the $X$-value our main consideration is that the minimum size of the order $Q-X=S-c$ should justify at least the ordering costs $a$. This leads to

$Q=X+\sqrt{2 a D / v r}$.

Now there are still a few things for which we must find a useful expression. The ordering frequency $f_{i}$ of item $i$ is set to $D_{i} / Q_{i}$. Then the average number of items, different from item $j$, that reach their order level during a period of length $R$ is found by

$F_{j}=R \sum_{i \neq j} f_{i}=R \sum_{i \neq j} D_{i} / Q_{i}$.

Under the assumption that the time between the placing of two orders of an item is exponentially distributed (which is perhaps not very realistic), we can easily calculate the probability $V$ that no items are ordered while the inventory level of item $j$ is in the interval $(s, s+X]$

$V=\frac{D / X}{D / X+F / R}=\frac{D}{D+X F / R}$.

An item can reach its order level because no other items are ordered while the inventory level is in the interval $(s, s+X]$, but also if the inventory level "jumps" below this interval. Of course, this depends on the probability distribution of the transaction size. If $X$ is small compared to the average transaction size $m_{1}$ the probability of such a jump $P_{\text {jump }}$ can be estimated by $1-X / m_{1}$. If $X$ is relatively big and this expression would yield a negative value, we set the probability $P_{\text {jump }}$ equal to 0 . Combining this probability and the probability $V$ we can now estimate the probability $P_{s}$ that an item reaches the order level $s$ or jumps below it:

$P_{s}=P_{\text {jump }}+\left(1-P_{\text {jump }}\right) V$. 
Now we can substitute Equations (5) and (8) in (4), such that we obtain a cost function containing $X$ as the only decision variable. The best $X$ value can be found very simply by evaluating the cost function for various $X$ values.

The last variable for which we have to find a proper value is the order level $s$. Under the assumption that the demand during the review period $R$ and the delivery period $L$ follows a normal distribution with parameters $\left(x_{R+L}, \sigma_{R+L}\right)$ in a single item situation a service level $P_{2}$ can be obtained if we choose the order level $s$ according to

$s=x_{R+L}+k \sigma_{R+L}+$ undershoot,

where $k$ is determined by

$G_{k}=\frac{Q\left(1-P_{2}\right)}{\sigma_{R+L}}$

with

$G_{k}=\int_{k}^{c}(u-k) \exp \left(-u^{2} / 2\right) / \sqrt{(2 \pi) \mathrm{d}(u)}$

a special function of the normal distribution [1]. For the average undershoot we have used their choice:

undershoot $=\frac{m_{2}}{2 m_{1}}-0.5$,

where $m_{i}$ is the $i$ th moment of the transaction size distribution. In the multi-item situation we obtain a much higher service level than required if we use an order level determined in this way. A simulation study showed that we could obtain quite accurate results if we tripled the shortage level $\left(1-P_{2}\right)$ for items with a $P_{s}$ value near to 0 , while the shortage level for items with a $P_{s}$ value near to 1 remained unchanged. We also adapted the undershoot for the probability $P_{s}$. Thus we obtained the following formulae.

$$
\begin{aligned}
& G_{k}=\frac{3}{\left(1+2 * P_{s}\right)} \frac{Q\left(1-P_{2}\right)}{\sigma_{R+L}}, \\
& s=x_{R+L}+k \sigma_{R+L}+P_{s} * \text { undershoot. }
\end{aligned}
$$

In order to find the best $X$ and $Q$ values we repeat the following steps a number of times, until the values do not change any more.
1. Determine $Q$ for every item, the first time according to the EOQ formula and later on according to (5).

2. Determine $F$ for every item according to (6).

3. Determine $P_{s}$ for every item according to (9).

4. Determine the best value for $X$ by evaluating (4).

Finally we determine $s$ by formula (14).

\section{Theoretical alternatives}

The first one to introduce an $(s, c, S)$ model for stochastic demand was Balintfy [4]. It has been a subject of many papers since then, but we will only mention a few of them. Only under very strong assumptions the values of $s, c$ and $S$ can be determined optimally [5]. Silver [6] assumed that the demand was Poisson distributed and developed an algorithm that determined $c$ and $S$ values for a situation with a negligible delivery time $L$ (therefore $s=0$ too !).

Later on, Silver [7] developed an inventory system that could deal with a compound Poisson demand. The same discrete demand process was considered by Federgruen et al. [2]. They give an algorithm that yields values for the control variables $s, c$ and $S$. The algorithm can be divided into two parts. In one subalgorithm an optimal $(s, c, S)$ strategy is determined for every item, using a Markov-Decision-Problem modelling with a given special ordering probability. This special ordering probability (Poisson distributed, independent of the inventory level) is determined in the other subalgorithm. Here we notice some similarities with our order frequency $F$. The special ordering probability indicates that one of the other articles from the same supplier will be ordered. This implies that with a certain probability we can order for the reduced ordering costs $a$. In this method the required service level is obtained by varying the imaginary shortage costs. Higher shortage costs yield a higher service level and vice versa.

Most of the methods for the stochastic multiitem inventory problem are two-step methods. They are based on a good interaction between solving the single item problem and the adaption of the single item parameters by considering the 
Table 3

Dataset characteristics

\begin{tabular}{llllllll}
\hline Item & $a$ & vr & $L$ & $\sigma(L)$ & $\lambda$ & $m_{1}$ & $\sigma$ (demand) \\
\hline 1 & 5 & 1 & 1 & 0.46 & 4 & 1 & 0.25 \\
2 & 5 & 1 & 1 & 0.46 & 4 & 1 & 1.00 \\
3 & 5 & 1 & 1 & 0.46 & 1 & 1 & 0.25 \\
4 & 5 & 1 & 5 & 1.73 & 1 & 1 & 0.25 \\
5 & 5 & 1 & 5 & 1.73 & 1 & 1 & 0.25 \\
6 & 5 & 1 & 5 & 1.73 & 1 & 4 & 1.00 \\
\hline
\end{tabular}

multi-item problem. Like in our simple model, this interaction is repeated until improvement stops.

In order to judge the performance of our simple model presented in Section 3 we considered six arbitrary datasets. By means of simulation we compared the performance of our model with the Federgruen et al. model. In the first set we have chosen $A=15$ and we consider six different items for which the characteristics such as the reduced ordering costs, the product of the price and the holding cost rate (per year), the average delivery time and its standard deviation, the average time between two demand transactions ( $\lambda$, in weeks), the average transaction size and its standard deviation are given in Table 3. The review period $R$ is taken to be one week.

The choices for $s, c$ and $S$ as they were made by the two methods are presented in Table 4.

The resulting performance, containing the service level (a level of 0.97 was required), the average number of item-orders per year, the average inventory level and the total costs per year, are presented in Table 5.
Table 4

Control parameters

\begin{tabular}{|c|c|c|c|c|c|c|}
\hline \multirow[t]{2}{*}{ Item } & \multicolumn{3}{|c|}{ Simple rule } & \multicolumn{3}{|c|}{ Federgruen rule } \\
\hline & $s$ & $c$ & $S$ & $s$ & $\mathcal{c}$ & $S$ \\
\hline 1 & 0 & 6 & 17 & 0 & 6 & 18 \\
\hline 2 & 0 & 6 & 17 & 0 & 8 & 22 \\
\hline 3 & 1 & 17 & 40 & 0 & 16 & 40 \\
\hline 4 & 5 & 21 & 44 & 2 & 22 & 46 \\
\hline 5 & 5 & 21 & 44 & 2 & 22 & 46 \\
\hline 6 & 20 & 59 & 104 & 23 & 71 & 118 \\
\hline
\end{tabular}

As we can see in this table, the performance of both methods is almost equal. In the other five datasets we noticed the same behaviour. The only difference seems to be that the simple rule yields slightly more orders and a lower average inventory level. In our opinion, the simple rule offers more insight to the purchasers because of its EOQ components than the Federgruen method or the Silver [7] method and it makes it also much easier to calculate the values of the control variables.

\section{Application of the simple model}

Before the model was taken into use, it was tested in a simulation study and the results were compared to the initial situation. In this study, we considered 1544 items, which represented more than $60 \%$ of the total inventory. For the other items there was either insufficient information or

Table 5

Performance comparison between simple rule and Federgruen rule

\begin{tabular}{|c|c|c|c|c|c|c|c|c|}
\hline \multirow[t]{2}{*}{ Item } & \multicolumn{4}{|c|}{ Simple rule } & \multicolumn{4}{|c|}{ Federgruen rule } \\
\hline & $P_{2}$ & Orders & Inventory & Costs & $P_{2}$ & Orders & Inventory & Costs \\
\hline 1 & 0.990 & 1.08 & 10.02 & 15.40 & 0.983 & 0.93 & 10.55 & 15.21 \\
\hline 2 & 0.975 & 1.15 & 10.12 & 15.88 & 0.983 & 0.95 & 12.91 & 17.63 \\
\hline 3 & 0.988 & 1.85 & 22.63 & 31.88 & 0.983 & 1.75 & 21.85 & 30.58 \\
\hline 4 & 0.987 & 1.86 & 22.36 & 31.69 & 0.976 & 1.75 & 23.20 & 31.93 \\
\hline 5 & 0.973 & 1.83 & 21.77 & 30.95 & 0.967 & 1.69 & 22.92 & 31.39 \\
\hline 6 & 0.950 & 2.73 & 45.17 & 58.81 & 0.978 & 2.40 & 56.71 & 68.73 \\
\hline total & 0.975 & 3.10 & 132.07 & 231.11 & 0.977 & 2.70 & 148.14 & 235.97 \\
\hline
\end{tabular}


Table 6

Final simulation

\begin{tabular}{lcccc} 
& \multicolumn{2}{c}{ Central warehouse } & \multicolumn{2}{c}{ Sterile warehouse } \\
\cline { 2 - 5 } & Simple rule & VOBE & Simple rule & 0.970 \\
\hline Service rate & 0.970 & 0.954 & 0.988 & 6040 \\
Item-orders & 3510 & 5430 & 3980 & 1090 \\
Supplier-orders & 1070 & 1820 & 43.000 & 65.000 \\
Ordering costs & 105.300 & 175.400 & 131.800 & 138.000 \\
Holding costs & 153.500 & 205.700 & 174.800 & 203.000 \\
Total costs & 258.800 & 381.100 & & VOBE \\
\hline
\end{tabular}

Table 7

Implementation Results

\begin{tabular}{|c|c|c|c|c|}
\hline & \multicolumn{2}{|c|}{ Before implementation } & \multicolumn{2}{|c|}{ After 1 year } \\
\hline & Service & Inventory & Service & Inventory \\
\hline $\mathrm{CW}-0$ & 0.939 & 500.400 & 0.966 & 512.400 \\
\hline CW-1 & 0.926 & 235.400 & 0.943 & 211.800 \\
\hline CW-2 & 0.899 & 49.200 & 0.942 & 42.000 \\
\hline CW-3 & 0.960 & 740.600 & 0.971 & 746.500 \\
\hline CW-8 & 0.941 & 33.600 & 0.949 & 39.600 \\
\hline
\end{tabular}

very unreliable information. Therefore, they were not taken into account in this comparison.

In the simulation we considered the performance for all items over 100 periods of one year, with Poisson arrival times and normally distributed transaction sizes. Using a geometrical distribution instead of a normal distribution for the transaction size yielded results which were not significantly different. In Table 6 we present the results of the simulation: the service level, the number of itemorders, the number of orders for suppliers, the ordering costs, the holding costs and the total costs. We simulated both with the VOBE control values, or their adapted values if these were more reliable, and with the control values obtained by the simple rule. Comparing the heuristically determined values of the parameters $F$ and $P_{s}$ with their simulation value showed differences which were usually in the region between 10 and $50 \%$. These differences and the gaps between estimated inventory levels and estimated order costs and the simulation results stimulated Quartel and Dellaert [8] to an improved (but slightly more complicated) rule.
The major results of this test are as follows:

- The service rate increases to the required level (CW) or almost to this level (SDW).

- The number of supplier-orders decreases with 35 to $40 \%$.

- The total inventory value and the corresponding holding costs decrease with $25 \%$ for the $\mathrm{CW}$ and $5 \%$ for the SDW.

- The total costs decrease with over Dfl. 150.000 (about 25\%).

Of course, the cost decrease in combination with the increase of the service rate is quite remarkable. Therefore, the hospital implemented this rule in their VOBE system. One year after the implementation in the central warehouses the service rates and inventory levels, obtained by practical measuring in each of the five warehouses, are as presented in Table 7.

The number of supplier-orders decreased with about $20 \%$ during this year. There are three reasons why these figures can even be better in future:

1. During the last year the demand value increased with about $20 \%$, therefore the values in the last column are higher than expected.

2. For a number of articles the control variables cannot be determined by the simple rule, because of insufficient or incorrect information. Therefore the overall service rate does not reach the 0.97 level.

3. The VOBE order advice is not always followed by the purchasers (they often prefer the previous order size), though much more than before.

Nevertheless, the hospital management was very much satisfied, especially with the service rate improvement and the purchasers now have order advises in which they have confidence. Of course, 
there are still items that need special attention and which cannot be treated by a general model like this one, but for the majority of items the simple model works very well.

\section{References}

[1] Silver, E.A. and Peterson, R., 1985. Decision systems for inventory management and production planning, second ed. Wiley, New York.

[2] Federgruen, A., Groenevelt, H. and Tijms, H.C., 1984. Coordinated replenishment in a multi-item inventory system with compound Poisson demands. Management Science, 30(3): 344-357.
[3] Horngren, C.T. and Foster, G., 1987. Cost accounting: a managerial emphasis, sixth ed. Prentice-Hall, Englewood Cliffs, N.J.

[4] Balintfy, J.L., 1964. On a basic class of multi-item inventory systems, Management Science, 10(2): 287-297.

[5] Ignall, E., 1969. Optimal continuous review policies for two product inventory systems with joint set-up costs. Management Science, 15(5): 278-283.

[6] Silver, E.A., 1974. A control system for coordinated inventory replenishment. Int. J. Prod. Res., 12(6): 647-671.

[7] Silver, E.A., 1981. Establishing reorder points in the $(S, c, s)$ coordinated control system under compound Poisson demand. Int. J. Prod. Res., 19(6): 743-750.

[8] Quartel, M. and Dellaert, N., 1994. Coordinated inventory control - an improved rule. Report 9459/A, Econometric Institute, Erasumus University Rotterdam. 